\title{
Osseous oligometastases from thymic carcinoma: a case report suggesting the effectiveness of palliative-intent radiotherapy treatment
}

This article was published in the following Dove Press journal:

OncoTargets and Therapy

29 February 2016

Number of times this article has been viewed

\author{
Jumpei Kashima' \\ Hirotoshi Horio' \\ Yusuke Okumal,2 \\ Yukio Hosomi' \\ Tsunekazu Hishima ${ }^{3}$ \\ 'Department of Thoracic Oncology \\ and Respiratory Medicine, Tokyo \\ Metropolitan Cancer and Infectious \\ Diseases Center Komagome Hospital, \\ Tokyo, Japan; ${ }^{2}$ Division of Oncology, \\ Research Center for Medical Sciences, \\ jikei University School of Medicine, \\ Tokyo, Japan; ${ }^{3}$ Department of \\ Pathology, Tokyo Metropolitan Cancer \\ and Infectious Diseases Center \\ Komagome Hospital, Tokyo, Japan
}

Background: Oligometastasis, a recently proposed concept, is defined as an intermediate state of cancer, between localized and systemic disease, that may be well controlled by local ablative treatment. Thymic carcinoma is a rare cancer with a poor prognosis. A definitive management approach has yet to be confirmed by a high level of evidence.

Case presentation: We present the case of a 41-year-old female who underwent curativeintent surgery for a stage III squamous cell carcinoma of the thymus. Bone metastases were detected 1 year later by magnetic resonance imaging. These were treated with palliative-intent radiotherapy. Disease progression has not been observed in more than 15 years since the achievement of complete radiological remission.

Conclusion: The treatment outcomes in this and other reported cases suggest that some patients with oligometastatic thymic carcinoma may achieve prolonged survival or even cure with lowdose radiotherapy delivered to the metastases.

Keywords: oligometastasis, thymic carcinoma, thymic epithelial tumor, bone metastasis

\section{Background}

The concept of oligometastasis was proposed by Hellman and Weichselbaum, ${ }^{1}$ who defined it as an intermediate state of cancer between locally limited disease and disease with distant metastases. Patients with oligometastases may have prolonged disease-free intervals or even be effectively cured following complete resection or ablation of their primary lesions and metastases. ${ }^{2}$ For example, a 10-year survival rate of approximately $20 \%$ was reported in patients undergoing resection of hepatic metastases from colorectal cancer together with control of the primary tumor site. ${ }^{3}$ Similar responses have been described following localized treatment of oligometastases of prostate, breast, and lung cancers. In the USA, retrospective and prospective studies aimed at assessing treatment efficacy in such cases are currently in progress. ${ }^{4}$

Thymic carcinoma is rare, and the lack of focal or systemic symptoms until the disease is in its advanced stage results in the poor prognosis of affected patients. ${ }^{5,6}$ The 5 -year survival rate is $80 \%$ for patients with Masaoka-Koga stage I/II disease, $63 \%$ for those with stage III disease, and $42 \%$ and $30 \%$ for those with stages IVa and IVb disease, respectively. ${ }^{7}$ By contrast, patients with thymoma typically present with immunological symptoms at an early stage. The optimal treatment for thymic carcinoma has yet to be confirmed by a high level of evidence because of the rarity of this cancer. In a single-institutional retrospective study of thymic carcinoma patients, we showed that staging and grade of atypia are significant prognostic factors, and that surgical intervention is associated with better survival. ${ }^{8,9} \mathrm{We}$ also found that the most common sites of $\mathrm{BY}$
hC for commercial use of this work, please see paragraphs 4.2 and 5 of our Terms (https://www.dovepress.com/terms.php). 
metastases from thymic carcinoma are the lung, lymph nodes, liver, and bone. ${ }^{8}$ Sasaki et al reported cases of two patients with thymic carcinoma and bone metastases. Both patients underwent chemotherapy and radiation of the bone lesions, resulting in 10- and $>18$-month survival. ${ }^{10}$

Here, we present the case of a thymic carcinoma patient whose disease has remained in remission for $>15$ years after palliative-intent radiotherapy for osseous oligometastases. Written informed consent was obtained from the patient for publication of this case report and the accompanying images and this report was reviewed and approved by Ethics committee of Tokyo Metropolitan Cancer and Infectious Diseases Center Komagome Hospital.

\section{Case presentation}

A 41-year-old female presented at the Tokyo Metropolitan Cancer and Infectious Disease Center Komagome Hospital (Tokyo, Japan), with chest pain. She had no previous medical history or a family history of heart disease. Serum levels of the tumor markers CEA, NSE, CA 19-9, and SCC were not elevated. A computed tomography scan revealed an anterior mediastinal tumor extending to the sternum, pericardium, mediastinal pleura, left upper lobe of the lung, and left brachiocephalic vein. Histopathologic examination of a computed tomographyguided biopsy revealed squamous cell carcinoma of the thymus (Figure 1). She underwent surgical resection of the thymus and the tumor-invaded surrounding organs, followed by reconstruction with a rectus abdominis musculocutaneous flap. The pathological diagnosis of the resected tumor confirmed thymic squamous cell carcinoma diagnosed after preoperative biopsy. Subsequently, the patient underwent postoperative chemoradiotherapy consisting of 60 Gy irradiation in 2 Gy fractions and one cycle of concurrent cytotoxic chemotherapy $(150 \mathrm{mg}$ of cisplatin on day 1 and $150 \mathrm{mg}$ etoposide $/ \mathrm{m}^{2}$ on days 1,3 , and 5). One year later, she presented with pain in her right foot joint. Magnetic resonance imaging showed round lesions in the distal part of the right tibia and the talus (Figure 2A and B). These lesions were approximately $12 \mathrm{~mm}$ in diameter, with rims of low signal intensity on T2-weighted imaging and enhancement following contrast administration. The diagnosis was therefore bone metastases. The initial plan was irradiation of the metastatic sites with $30 \mathrm{~Gy}$, delivered in 2 Gy fractions, but because of persistent ankle pain, the patient was treated with a boosted dose of $50 \mathrm{~Gy}$ total irradiation, delivered in $2 \mathrm{~Gy}$ fractions. Bone scintigraphy performed 8 years after radiation therapy showed that the bone lesions had diminished in size (Figure 2C), and that there was no remnant of the tumor. In more than 15 years since the resection of the patient's primary tumor, there have been no further symptoms, evidence of other metastases, or local recurrence.

\section{Discussion}

The term "oligometastasis" is used to describe a transitional or intermediate state between localized cancer and systemic disease. Experience has shown that patients with oligometastases can achieve prolonged progression-free intervals or even cure when treated by surgical resection or radiation of the tumors. ${ }^{2}$ However, studies of oligometastases are potentially biased by patient selection because these lesions are characteristically identified by imaging in patients undergoing regular and thorough follow-up, i.e., before wide-spread metastatic lesions have developed. Nonetheless, preclinical study has suggested that tumor cells from oligometastases are unable to metastasize to other organs. ${ }^{11}$ Thus, local control of oligometastases not only reduces tumor burden and improves organ function but also prevents the
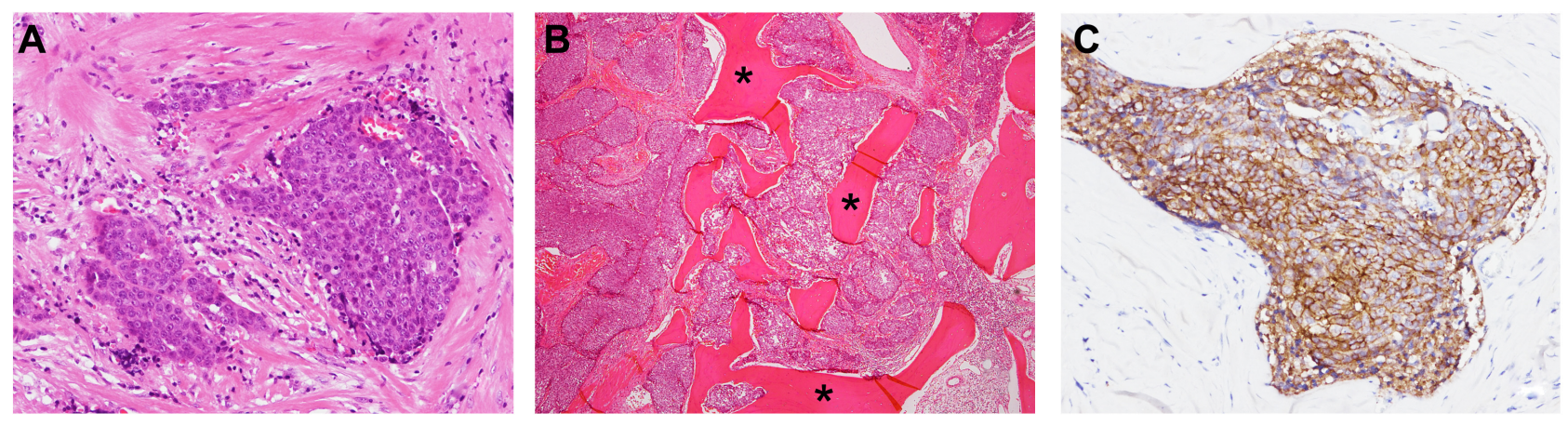

Figure I Pathological findings of the resected specimen.

Notes: The image shows the focal proliferation of cuboidal atypical cells with round nuclei and prominent nucleoli surrounded by fibrous tissue. Keratinization, plasmodesmata, and glandular construction are absent (A). Tumor invasion of the sternum (B). Immunohistochemical staining for KIT protein was positive in nesting tumor cells (C). *Bone trabeculae. 

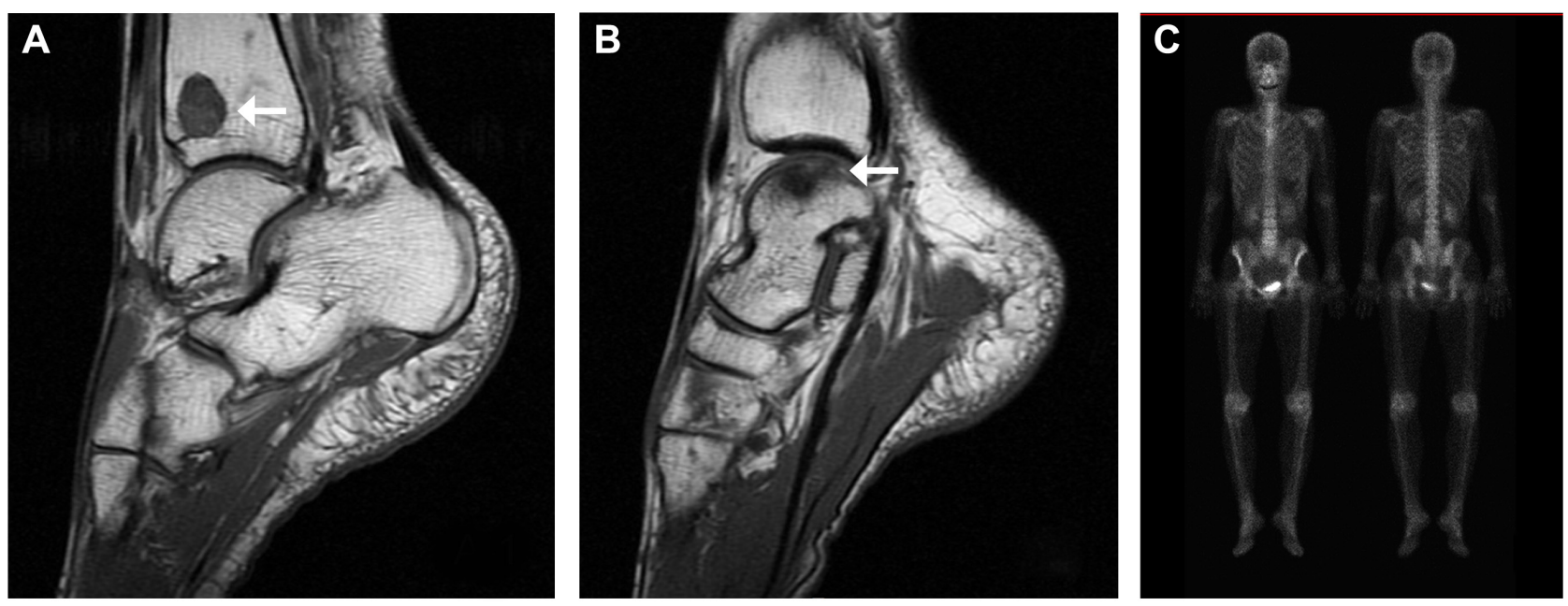

Figure 2 Osseous metastases presenting as foot joint pain.

Notes: Magnetic resonance imaging performed after the patient developed joint pain shows bone lesions (low-signal areas are indicated by arrows) in the right tibia (A) and talus (B). Bone scintigraphy shows no obvious lesion 8 years after palliative radiotherapy (C).

genetic evolution of the tumor cells, thereby delaying the development of more aggressive metastases.

Organ-specific symptoms are less frequently produced by thymic carcinoma than by thymoma, and most patients with thymic carcinoma do not develop immunological manifestations such as myasthenia gravis. ${ }^{6,7}$ This explains, at least in part, why patients with thymic carcinoma tend to present with advanced-stage disease. Moreover, the effectiveness of the treatment of thymic carcinoma has not been proven with a high level of evidence because this tumor is even rarer than the highly uncommon thymoma. Thus, to obtain a larger sample size and to share patient experience, thymic carcinoma and its treatment are being investigated in international collaborations and with the aid of case reports.

The clinical management of thymic carcinoma is similar to that of thymoma. Our patient was initially diagnosed with an undifferentiated carcinoma of the thymus; however, pathological review indicated a squamous cell carcinoma of the thymus, based on the 2015 World Health Organization classification. ${ }^{12}$ Patients with metastatic or recurrent thymic carcinoma are typically treated with chemotherapy or palliative-intent radiotherapy. Platinum- and anthracycline-based chemotherapies are mostly chosen and dose of curative-intent radiotherapy is typically $50 \mathrm{~Gy}$ or more. However, there is no high-level evidence supporting these treatment strategies as optimal.

Our patient underwent curative-intent surgery and concurrent postoperative chemoradiotherapy. Osseous metastases to the tibia and talus were found 1 year later. Irradiation of the metastatic sites with 50 Gy appears to have controlled the disease, as there has been no evidence of progression during the past 15 years. This is the first report of a progression-free interval of such lengthy duration after the treatment of oligometastases. In a previous report, we described a patient with thymic carcinoma and brain and pulmonary metastases in whom a long survival time was achieved with palliativeintent radiotherapy and chemotherapy. ${ }^{13}$ Chemotherapy and radiation were used to treat the primary and metastatic sites, with local control achieved for $>8$ years. However, the patient died of pulmonary metastases despite six cycles of chemotherapy, as his brain metastases could not be controlled, nor his previously irradiated pulmonary metastases. These two cases together suggest that oligometastases of thymic carcinoma respond well to radiation therapy, which may considerably prolong the survival of these patients.

\section{Conclusion}

Although reports on oligometastases may be vulnerable to selection bias, the outcome of the patient described in this report, who was treated by extended surgical resection of the primary tumor and palliative-intent irradiation of the osseous metastases, may encourage the local treatment of oligometastases from thymic carcinoma.

\section{Acknowledgment}

The authors would like to thank Enago (www.enago.jp) for the English language review. No specific funding was received for this work.

\section{Author contributions}

All authors contributed toward data analysis, drafting and revising the paper and agree to be accountable for all aspects of the work. 


\section{Disclosure}

The authors report no conflicts of interest in this work.

\section{References}

1. Hellman S, Weichselbaum RR. Oligometastases. J Clin Oncol. 1995; 13(1):8-10.

2. Macdermed DM, Weichselbaum RR, Salama JK. A rationale for the targeted treatment of oligometastases with radiotherapy. J Surg Oncol. 2008;98(3):202-206.

3. Sheth KR, Clary BM. Management of hepatic metastases from colorectal cancer. Clin Colon Rectal Surg. 2005;18(3):215-223.

4. Reyes DK, Pienta KJ. The biology and treatment of oligometastatic cancer. Oncotarget. 2015;6(11):8491-8524.

5. Ahmad U, Yao X, Detterbeck F, et al. Thymic carcinoma outcomes and prognosis: results of an international analysis. J Thorac Cardiovasc Surg. 2015;149(1):95-100.

6. Kuo T. Cytokeratin profiles of the thymus and thymomas: histogenetic correlations and proposal for a histological classification of thymomas. Histopathology. 2000;36(5):403-414.

7. Suster S, Rosai J. Histology of the normal thymus. Am J Surg Pathol. 1990;14(3):284-303.
8. Okuma Y, Hosomi Y, Takahashi S, Maeda Y, Okamura T, Hishima T. Response to cytotoxic chemotherapy in patients previously treated with palliative-intent chemotherapy for advanced thymic carcinoma. Clin Lung Cancer. 2015;16(3):221-227.

9. Quintanilla-Martinez L, Wilkins EW Jr, Choi N, Efird J, Hug E, Harris NL. Thymoma. Histologic subclassification is an independent prognostic factor. Cancer. 1994;74(2):606-617.

10. Sasaki S, Fukushima T, Maruyama Y, et al. Two Cases of Thymic Carcinoma Initially Presenting as Bone Metastasis: A Clinical Report and the Usefulness of CD5 Immunohistochemistry for Assessing Bone Lesions. Intern Med. 2015;54(14):1781-1785.

11. Weichselbaum RR, Hellman S. Oligometastases revisited. Nat Rev Clin Oncol. 2011;8(6):378-382.

12. Travis WD, Brambilla E, Burke AP, Marx A, Nicholson AG, editors. WHO Classification of Tumours of the Lung, Pleura, Thymus and Heart. 4th ed. Lyon: 2:154-298, 2015.

13. Nagamata M, Okuma Y, Yamada Y, Hosomi Y, Hishima T. Crucial role of treatment with palliative intent for a patient with advanced thymic carcinoma. Oncol Lett. 2014;8(2):513-516.
OncoTargets and Therapy

\section{Publish your work in this journal}

OncoTargets and Therapy is an international, peer-reviewed, open access journal focusing on the pathological basis of all cancers, potential targets for therapy and treatment protocols employed to improve the management of cancer patients. The journal also focuses on the impact of management programs and new therapeutic agents and protocols on

\section{Dovepress}

patient perspectives such as quality of life, adherence and satisfaction The manuscript management system is completely online and includes a very quick and fair peer-review system, which is all easy to use. Visit http://www.dovepress.com/testimonials.php to read real quotes from published authors. 